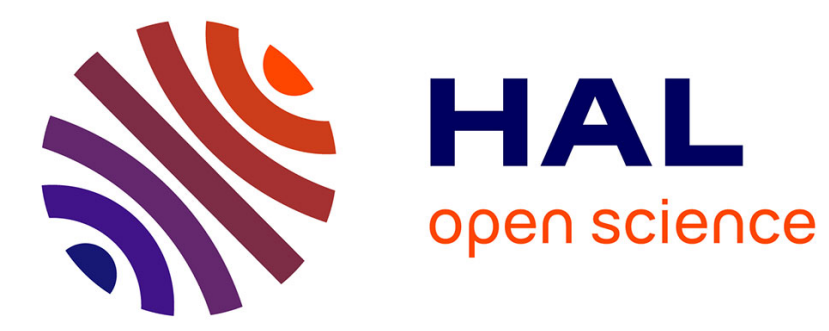

\title{
Optical properties of multilayered porous silicon
}

\author{
S. Setzu, Patrick Ferrand, R. Romestain
}

\section{To cite this version:}

S. Setzu, Patrick Ferrand, R. Romestain. Optical properties of multilayered porous silicon. Materials Science and Engineering: B, 2000, 69-70, pp.34-42. 10.1016/S0921-5107(99)00261-5 . hal-00272406

\section{HAL Id: hal-00272406 https://hal.science/hal-00272406}

Submitted on 26 Sep 2015

HAL is a multi-disciplinary open access archive for the deposit and dissemination of scientific research documents, whether they are published or not. The documents may come from teaching and research institutions in France or abroad, or from public or private research centers.
L'archive ouverte pluridisciplinaire HAL, est destinée au dépôt et à la diffusion de documents scientifiques de niveau recherche, publiés ou non, émanant des établissements d'enseignement et de recherche français ou étrangers, des laboratoires publics ou privés. 


\title{
Optical properties of multilayered porous silicon
}

\author{
S. Setzu a,b, P. Ferrand ${ }^{a}$, R. Romestain ${ }^{\mathrm{a}, *}$ \\ ${ }^{a}$ Laboratoire de Spectrométrie Physique, Université Joseph Fourier - CNRS (UMR 5588) B.P. 87, 38402 St Martin d'Hères Cedex, France \\ ${ }^{\mathrm{b}}$ Consorzio PROMEA-INFM, Sezione di Cagliari -Dipartimento di Fisica, Citt. Universitaria, 09042 Monserrato (CA), Italy
}

\begin{abstract}
We present a short review of some optical devices based on multilayered porous silicon, which can be easily obtained by varying the formation current during the etching process. These include Bragg reflectors and Fabry-Pérot microcavities, which can be adjusted from the visible to the near infrared. The interface roughness, tragic in the case of multilayers, is studied. It can be drastically reduced when changing the electrolyte viscosity. The high reflectivities obtained in this way are measured by Cavity Ring-Down Spectroscopy. Problems occurring when realising thin layers and an efficient way to adjust precisely the optical thicknesses of the thin layers constituting the multilayered structure are also presented. Finally we present a method of calculation of the emission which takes absorption into account and is able to explain the angular dependence of the luminescence. (C) 2000 Elsevier Science S.A. All rights reserved.
\end{abstract}

Keywords: Porous silicon; Microcavity; Roughness; Photoluminescence; Emission; Calculation

\section{Introduction}

The observation by Canham [1] of visible luminescence from Porous Silicon (PSi) definitely triggered a vast amount of studies on this material. However many of its properties were already well known. Namely its optical properties were studied by Pickering et al. [2] as early as 1984 and it was noticed that PSi could form flat dielectric layers of good optical quality. But it was only in 1994 that Vincent [3] proposed to use the easy modulation of the index by modulating the formation current to create superlattices and measured the reflectivity of such samples in the IR and visible range. In this paper we will give some examples of what can be achieved with such structures referring the reader to the very detailed review of Pavesi [4]. We will present the problems raised by fabrication and characterisation and their possible solutions. Finally we will discuss the emission properties of these structures, presenting a formalism which takes absorption into account and explains the angular dependence of the luminescence.

In order to achieve the maximum index modulation, $\mathrm{p}^{+}$type doped substrates are much better than $\mathrm{p}$ type. Typically $\mathrm{p}^{+}$porosity can range between 25 to $80 \%$

\footnotetext{
* Corresponding author. Tel.: + 33-4-76514726, Fax: +33-476514544.

E-mail address: romestai@spectro.ujf-grenoble.fr (R. Romestain)
}

yielding $n$ values between 3 and 1.4 (at $800 \mathrm{~nm}$ ) whereas p type only corresponds to indices between 1.9 and 1.2. One must notice that the maximum porosity attainable in thick layers before samples crack upon drying can be overcome since only very thin layers are stacked between more robust layers. In addition $\mathrm{p}^{+}$substrates in usual conditions give rise to much smoother interfaces than $\mathrm{p}$ substrates, inducing less scattering of light [5]. However $\mathrm{p}$ type substrates lead to less bulk absorption and display much brighter luminescence. For both types one has the possibility of decreasing significantly the absorption by oxidising the whole structure [6].

The most simple structure is undoubtedly the wave guide, which in its simplest form requires only two layers, since it will be dealt with in a following paper we will refer the reader to [7]. Periodic layers of alternating high index $n_{1}$ (thickness $t_{1}$ ) and low index $n_{2}$ (thickness $t_{2}$ ) lead to the well known distributed Bragg reflectors (DBR). They display a plateau of high reflectivity, which can be viewed as 1D photonic band gap, centred at $\lambda=2\left(n_{1} t_{1}+n_{2} t_{2}\right)$. The width of the high reflectivity plateau is determined by the ratio of the indices. It has been enlarged by changing the value of the centre $\lambda$ during the growth of the structure [8]. This occurs at the expense of the maximum reflectivity. Another clever tailoring of the optical response has been realised by performing a sine modulation of the index instead of a step modulation [9]. Such a 'rugate' filter is also charac- 
terised by a strong reflectance domain but with the absence of the side lobes typical of a DBR of finite length. If the index modulation consists of the superposition of two sine waves then two maxima of reflectivity are obtained [9]. A very ingenious improvement of the DBR has been realised by Hukel et al. [10]. They induce a voltage drop along the substrate so modifying the electrical characteristics laterally while forming the sample. This results in a laterally varying wavelength of optimum reflection, typically from 750 to $500 \mathrm{~nm}$ on the same sample.

DBR's can be used to realise optical microcavities by inserting a $\lambda / 2$ layer called a spacer. It was demonstrated as early as 1995 using an all PSi structure by Pavesi et al. [11] on a hybrid structure where the top mirror is a $25 \mathrm{~nm} \mathrm{Ag}$ film [12]. Due to inhomogeneities in the nanostructure, the PSi luminescence is very broad (typically $0.4 \mathrm{eV}$ or more). The effect of the microcavity is to drastically reduce the emission width. However one has to keep in mind that what is not detected in a particular geometry will actually be emitted in another direction where the cavity is matched. An important problem is due to the fact that both the cavity layer and the DBR mirrors emit. Their emission is not modified the same way as the real cavity and the whole emission looks like a sharp peak on top of a wide background. Even in this case the oscillator strength of the emission is spread over a broad band and the coupling is called weak. This is not the case for the IR lines due to vibrations of the $\mathrm{Si}-\mathrm{H}$ bonds covering the surface of PSi, the linewidth being smaller than $20 \mathrm{~cm}^{-1}$. Fig. 1 shows clear evidence [13] of a strong coupling where the cavity mode is actually mixed with the molecular vibra-

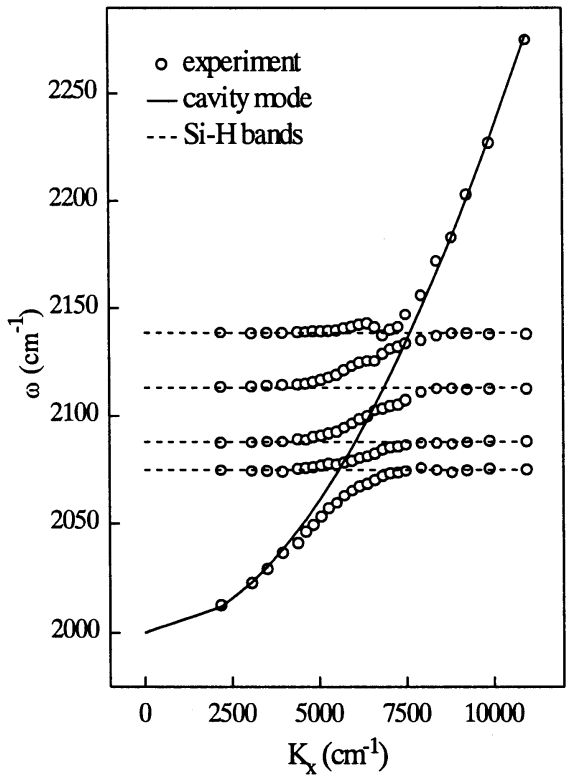

Fig. 1. Dispersion curves for the coupled mode between a PSi cavity and $\mathrm{Si}-\mathrm{H}$ vibrations [13]. tion and consequently frequencies are repelled from each other instead of crossing.

The amplification of the electromagnetic wave can also lead to interesting phenomena such as exaltation of the Raman effect which has been seen in III-V structures [14] or second harmonic generation. This last phenomena has been reported recently but with a simple DBR structure [15], the observed efficiency is presumably due to phase matching. The wave vector of the DBR can compensate the mismatch between the propagation $\mathbf{k}_{2}$ and the generation $2 \mathbf{k}_{1}$ wave vectors of the second harmonic.

\section{Experimental details}

The PSi samples that we studied were realised by electrochemical anodisation in the dark. We used single-crystal $7 \Omega . c m$ p type (100)-oriented Si substrates. For the sample realisation we used a solution $\mathrm{HF}: \mathrm{H}_{2} \mathrm{O}:$ Ethanol $=35: 35: 30$. In both the cases of single and multilayers the anodisation current densities we used were $16.6 \mathrm{~mA} / \mathrm{cm}^{2}$ and $166 \mathrm{~mA} / \mathrm{cm}^{2}$. The values of the $\mathrm{PSi} /$ crystalline $\mathrm{Si}$ interface roughness were determined by means of a Tencor P10 profilometer after the dissolution of the PSi layer. The reflectivity and transmission spectra were obtained by means of a PerkinElmer Lambda 9 spectrophotometer. The determination of the reflectivity coefficients of the DBR has been performed by cavity ring-down spectroscopy (CRDS) measurements using a pulsed Nd:YAG laser $(1.06 \mu \mathrm{m})$ and a $\mathrm{PbS}$ cell detector.

The single-layer and the microcavity presented in Fig. 10 are filled with a $10^{-2} \mathrm{M}$ solution of Rhodamine 800 in ethanol. The impregnation time depends on the porosity and on the surface state of the PSi. The PSi samples used as matrix were thermally oxidised at $450^{\circ} \mathrm{C}$ and then immersed in a Rhodamine 800 in ethanol solution at room temperature for $10 \mathrm{~s}$. The samples are then dried up under a $\mathrm{N}_{2}$ flux and all experiments were performed on dry samples.

Photoluminescence (PL) measurements are made exciting the sample with the green line $(514.5 \mathrm{~nm})$ of an $\mathrm{Ar}^{+}$ion laser, through a multimode optical fibre fixed on a rotating sample holder, with an incidence angle of $20^{\circ}$. The pumping beam is not polarised. The emitted light is analysed in a Chromex monochromator equipped with a $80 \mathrm{~g} / \mathrm{mm}$ grating, and acquired by a Princeton $512 \times 512$ channels charge coupled device (CCD). The spectral resolution is better than $1 \mathrm{~nm}$. An iris diaphragm with a diameter of $5 \mathrm{~mm}$ placed in front of the collecting lens selects an angular aperture of $1.4^{\circ}$. The power density of excitation is typically $50 \mathrm{~mW} / \mathrm{cm}^{2}$ and the acquisition time is $1 \mathrm{~s}$. Data are corrected by a geometric factor to avoid the increasing of surface area seen by the detector while tilting. 


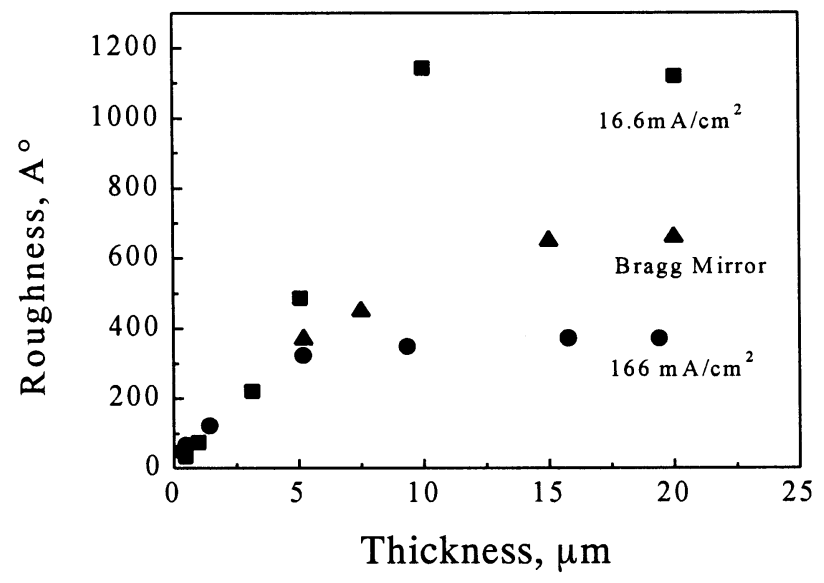

Fig. 2. We report here the roughness of layers obtained with two current densities: $16.6 \mathrm{~mA} / \mathrm{cm}^{2}(\boldsymbol{\square})$ and $166 \mathrm{~mA} / \mathrm{cm}^{2}(\bullet)$. The corresponding refractive indices are 1.7 and 1.3 respectively. The final value of the roughness for the DBR (triangles) is also shown. These were composed of 20,30,60 and 90 periods, with total thicknesses of $5,7,15$ and $20 \mu \mathrm{m}$ respectively. All samples were fabricated at room temperature.

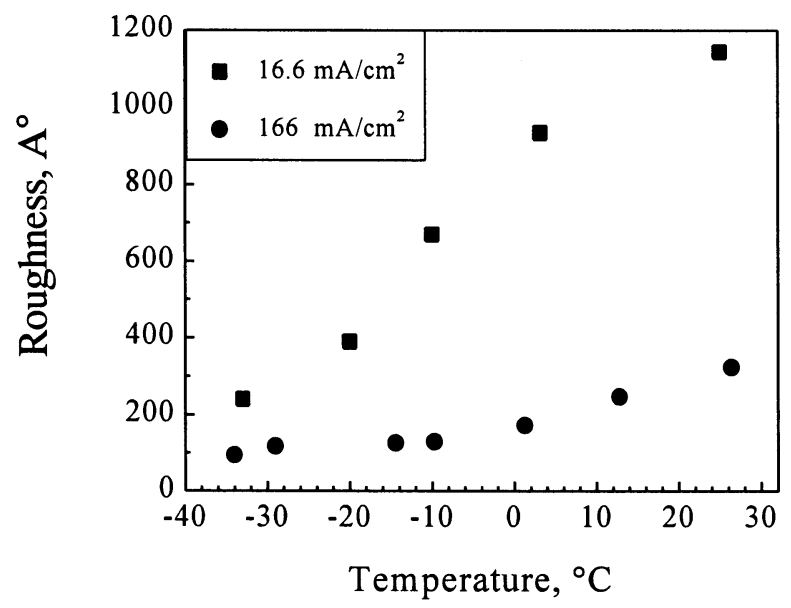

Fig. 3. A strong reduction of the roughness can be obtained by lowering the formation temperature. The measurements were realised on $10-\mu \mathrm{m}$ thick samples.

\section{Results}

\subsection{Interface roughness}

Due to the multiple reflections inside the multilayered structure, the quality of the interfaces is a crucial parameter. The evolution of the roughness as a function of the layer thickness for different current densities is known [5]: it is initially linear and then saturates. In Fig. 2 we show the evolution of the roughness as a function of the layer thickness for two current densities. The lower current density induces a roughness up to three times higher (for a $20-\mu \mathrm{m}$ thick layer) than that induced by the higher current density. Every point on the graph corresponds to a different sample. All sam- ples are realised on substrates with a surface roughness of about $7 \AA$.

In the case of a multilayered structure there is a periodic alternation of two current densities. Every time we change the current density a new porous layer will be created starting from the last interface. This interface will have a different roughness at each current change. The important point is now to understand the evolution of the roughness as a function of the sample thickness, for instance when realising a DBR. The roughness in every layer can just add to one another, or the applied current densities can lead eventually to their own roughness limit. We realised four DBR with different numbers of periods and total thicknesses. The final layer roughnesses measured after dissolution of the PSi layers are shown as triangles in Fig. 2.

We can see that the values of the roughnesses of the different DBR are between the two limit values obtained for simple layers with the same thickness and realised with the two current densities we are considering. Moreover, from the measured value of the multilayers roughness we can deduce that this is related to the relative value of the thickness of the two layers constituting the multilayered structure, according to

$\sigma_{\mathrm{DBR}}(e)=\left[t_{1} \sigma_{1}(e)+t_{2} \sigma_{2}(e)\right] /\left(t_{1}+t_{2}\right)$,

where $\sigma_{\mathrm{DBR}}(e), \sigma_{1}(e)$ and $\sigma_{2}(e)$ are the roughness values of samples with a final thickness $e$, respectively for a DBR (of period $t_{1}\left(I_{1}\right)+t_{2}\left(I_{2}\right)$ ) and simple layers realised with current densities $I_{1}$ or $I_{2}$. In our case, $\sigma_{\mathrm{DBR}}(e)$ is given by $\left[2 \sigma_{1}(e)+3 \sigma_{2}(e)\right] / 5$.

If we then decrease the values of $\sigma_{1}$ and $\sigma_{2}$ we will decrease the value of $\sigma_{\mathrm{DBR}}$. This can be realised by controlling the formation temperature of the PSi layer. Fig. 3 reports the values of the saturating interface roughness as a function of the formation temperature for thick samples obtained with two current densities. For the lower current density we obtained a reduction of the roughness as a function of temperature up to a factor of six by changing the temperature from +26 to $-35^{\circ} \mathrm{C}$. For the higher current density the decrease is by a factor of three for the same temperature variation. If we use the Eq. (1), we obtain that at $26^{\circ} \mathrm{C}$ the roughness of a multilayer $10 \mu \mathrm{m}$ thick is $600 \AA$ and that at $-35^{\circ} \mathrm{C}$ is about $150 \AA$. This means that there is a decrease in the Bragg mirror roughness by a factor of four. The Davies-Bennett relation [16,17], that is also valid for the PSi, directly relates the light diffusion to the interface roughness $\sigma, D=R_{0}(1-\exp (-(4 \pi \sigma)$ $\left.\lambda)^{2}\right)$ ) where $R_{0}$ is the reflection coefficient for a perfectly plane surface, and $\lambda$ is the wavelength. At $1200 \mathrm{~nm}$, the light diffusion decreases in the case of the DBR by a factor 13.5. This allows the realisation of high quality multistructures with reflectivity coefficients up to $99.64 \%$ for the DBR and a typical full width at half maximum (FWHM) of the cavity mode of $5 \mathrm{~nm}$, as 
shown in Fig. 9. More details on the influence of the formation temperature on the PSi are given elsewhere [18].

This decrease of the roughness by lowering the temperature can be explained by the increase of the viscosity of the electrolytic solution. Let us consider the critical electropolishing current as limit situation for no layer roughness. In this condition it is generally admitted that a viscous state exists near the etched surface in which the diffusion of the reactive chemical species is difficult [19]. In this regime the electrochemical reaction is favoured on the peaks and has then a smoothening role. Turner [19] has described the role of the viscosity of the solution on the value of the critical electropolishing current. In particular, an increase in the viscosity decreases the critical current value. On this basis we can infer that by increasing the viscosity of the electrolytic solution we should obtain smoother PSi layers.
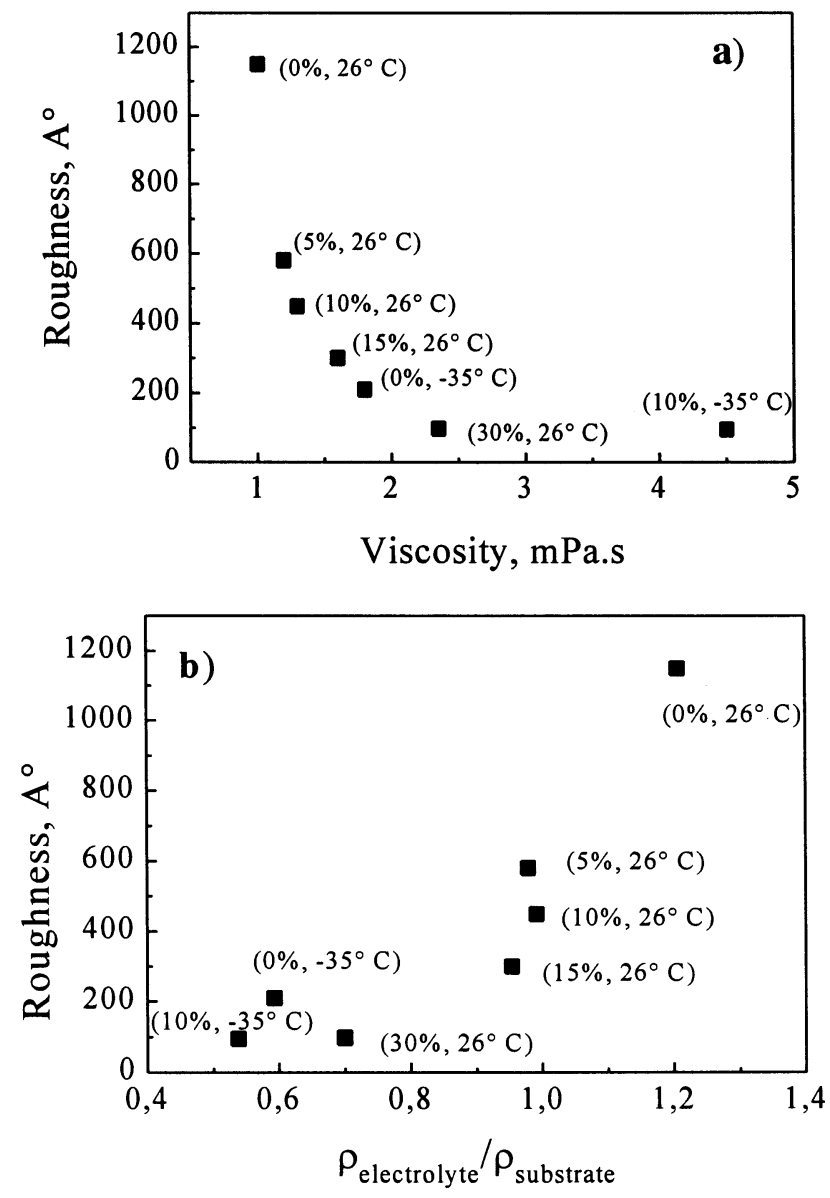

Fig. 4. Evolution of the roughness as a function of the solution viscosity (a) and ratio of resistivities (b). All samples studied are 10 $\mu \mathrm{m}$ thick and anodised with a current density of $16.6 \mathrm{~mA} / \mathrm{cm}^{2}$. On each point side we report the glycerol concentrations and the formation temperature. The viscosity of the solutions has been determined by analysing the fall of a sphere in a tube filled with the solution. The relation between the resistivity and the roughness is not unique, e.g. the same resistivity value can correspond to three different roughness values.

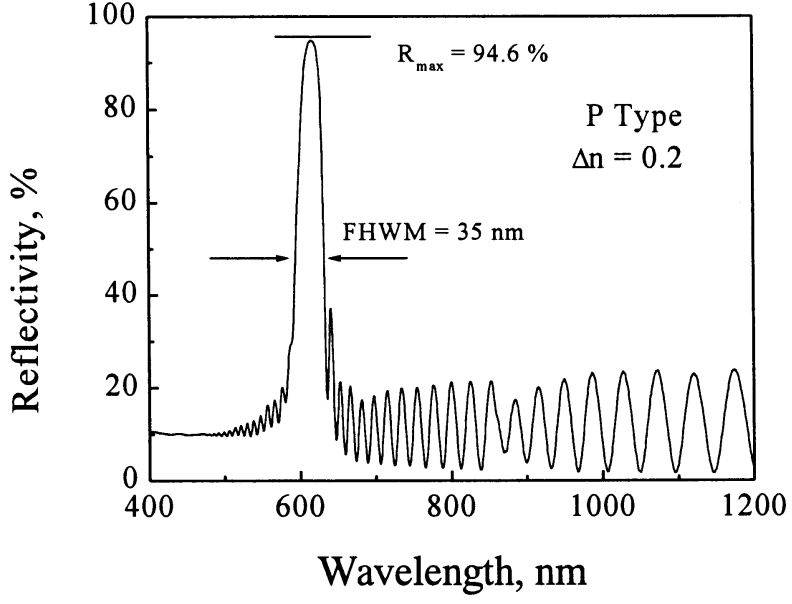

Fig. 5. It is possible to realise a DBR with a $35 \mathrm{~nm}$-wide forbidden band using a small refractive index variation. In this case the mirror is composed of 40 periods and the values of the refractive indices are $n_{1}=1.9$ and $n_{2}=1.7$ for the two layers.

A different approach, proposed by Chazalviel et al. [20] is to explain the smoother interface by electrostatic effects through the increase of the ratio between the resistivities of the substrate and of the electrolyte. They show that for the PSi formation regime to be more stable (smoother) the electrolyte resistivity should be larger than that of the substrate.

We measured the resistivity and viscosity of several electrolytic solutions at two temperatures (26 and $35^{\circ} \mathrm{C}$ ) and with different glycerol contents. With these solutions we realised several thick samples and we measured their roughness. In Fig. 4 we show the evolution of the interface roughness as a function of (a) the solution viscosity and (b) the resistivity of the electrolyte. It appears from the comparison between Fig. 4a and $b$ that there is a stronger correlation between roughness and viscosity than for roughness and resistivity. All experimental values for Fig. 4 a lies on one curve while for Fig. $4 b$ we can have different roughness values for a given resistivity.

\subsection{Accurate DBR characterisations}

Usually a broad reflection plateau is looked for and realised with large index ratios. Narrow band filters can also be useful. As an example, a DBR with a large number of periods (40) and small $\Delta n(0.2)$ realised at $T=-35^{\circ} \mathrm{C}$ is shown in Fig. 5. This kind of mirror, that can be useful as a narrow band filter, shows a FWHM of the reflectivity stop band of $35 \mathrm{~nm}$ and a maximum reflectivity $R_{\max }=94.6 \%$. This value of $R_{\max }$ has been determined by standard reflectivity.

However, when realising a DBR with larger $\Delta n$ we increase both the FWHM and $R_{\max }$ of the reflectivity stop band. In such structures it is possible to obtain $R_{\max }$ values larger than $99 \%$ and the standard reflectiv- 
ity is no more adapted to a precise measurement. In this case it is more appropriate to realise the structure on double side polished substrates, that allow transmission measurements (Fig. 6). The comparisons of reflectivity and transmission spectra give an evaluation of the losses, and the value of $R$ can be calculated more precisely. In the case of extremely high reflectivities $(R>99.5 \%)$ even this technique becomes unsuitable.

For this reason we also performed cavity ring-down spectroscopy (CRDS) measurements. The CRDS is a highly sensitive and versatile technique for low absorption measurements [21]. A ring-down cavity is generally composed of two dielectric high reflectivity mirrors aligned at a distance $D$ in such a way that an optical resonator is formed. A light pulse is injected in a ring-down cavity through one of the mirrors. Even if

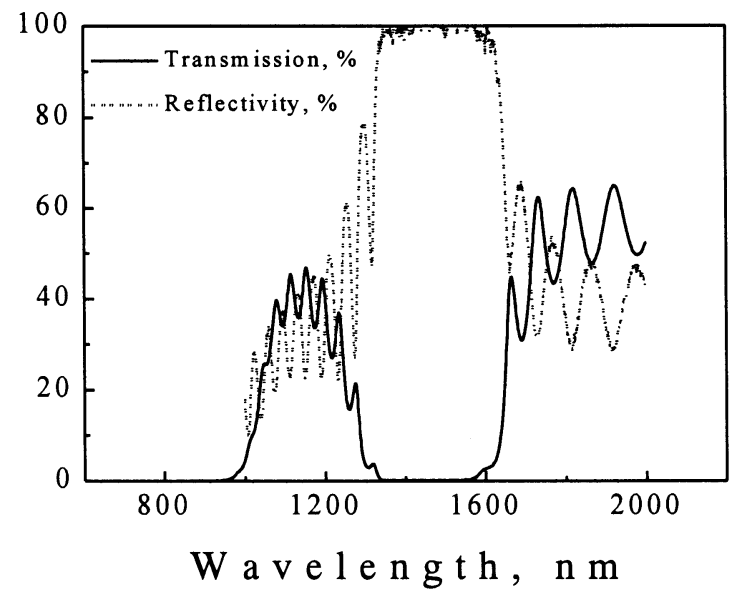

Fig. 6. Transmission (solid line) and reflectivity (dotted line) spectra of a 20 period DBR realised on a both-side polished substrate. The transmission (dashed) of the $550-\mu \mathrm{m}$ thick crystalline $\mathrm{Si}$ substrate is also shown.

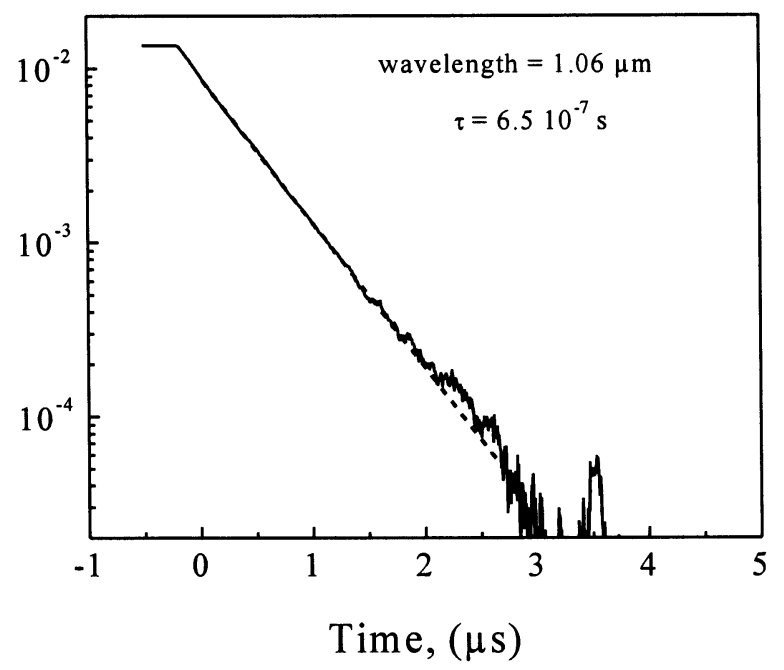

Fig. 7. Ring down signal as a function of time (solid line) for a 24-h period DBR centred at $1.06 \mu \mathrm{m}$. The signal can be fitted (dashed line) with a first order decay. the transmission may be weak (in the visible, for an optimal dielectric mirror, it can be lower than $10^{-5}$ ) the pulse fraction injected can still consist in more that $10^{10}$ photons and generates detectable signal after transmission through the other mirror. This ring-down signal has an envelope that is simply a first order decay of the $\exp (-t / \tau)$ type. When the reflection coefficient $R_{1}$ of the first mirror is $99.99 \%$ one can derive the lifetime of the photons in the cavity $\tau$ as $\tau^{-1}=c\left(1-R_{2}+\alpha D\right) / 2 D$ where the absorption $\alpha$ of the medium (air in our case) is very low and $R_{2}$ is the reflection coefficient of the second mirror.

If as a second mirror we use a PSi DBR realised on a two-sides-polished Si substrate and centred at 1.06 $\mu \mathrm{m}$ we can, starting from the time constant $\tau$, determine its reflection coefficient with a precision of $0.01 \%$. The ring-down signal is shown in Fig. 7. The fit of the ring-down signal allows the determination of the time constant $\tau=6.5 \times 10^{-7} \mathrm{~s}$. Because our cavity is $35 \mathrm{~cm}$ long, we obtain a reflection coefficient for our DBR of $99.64 \%$. It is possible to use this technique only for microcavities or DBR's centred at wavelengths larger than the silicon gap wavelength $(1.1 \mu \mathrm{m})$.

\subsection{Formation parameters for multilayers}

The easiest way for realising a multilayer structure is to determine the dissolution rate and refractive index on thick $(\approx 3 \mu \mathrm{m})$ samples and use these values to calculate the thickness of the thin layers. Unfortunately, this method showed to be incorrect. As matter of fact, DBR's realised in this way always show a resonance wavelength larger than expected. This implies that the optical thickness of the DBR period is larger than $\lambda / 2$. To understand these results we realised a series of microcavities whose DBR periods are composed of two layers with refractive indices $n_{1}$ (top layer) $>n_{2}$ and whose spacer has a refractive index $n_{\mathrm{S}}=n_{2}$ and a thickness $t_{\mathrm{S}}=2 t_{2}$. We tuned the ratio $\delta=n_{1} t_{1} / n_{2} t_{2}$ by varying the formation times of the two kind of layers on each sample up to a factor of three (while keeping constant the resonance wavelength). The optical measurement in the TE and TM polarisation modes (Fig. 8) revealed that there are two distinct $\lambda_{\mathrm{TE}}$ and $\lambda_{\mathrm{TM}}$ cavity resonance wavelengths, whose spectral position depends on the detection angle $\theta$. The separation of the peaks, $\Delta \lambda=\lambda_{\mathrm{TE}}-\lambda_{\mathrm{TM}}$, can be as large as $25 \mathrm{~nm}$. The two values can coincide, if possible, only for one given value of $\theta$, as is the case for sample fp 81 in Fig. 8. When the two optical thicknesses are equal so that the two peaks coincide at non-zero angle. By fitting the positions of the resonances as a function of $\theta$, we were able to determine the values of $\delta$ for each sample. In order to fit the angular dependence of $\lambda_{\mathrm{TE}}$ and $\lambda_{\mathrm{TM}}$ for the sample realised using the conditions determined on thick layers (sample fp80) we need to use $n_{1} t_{1}=1.5 n_{2} t_{2}$. 


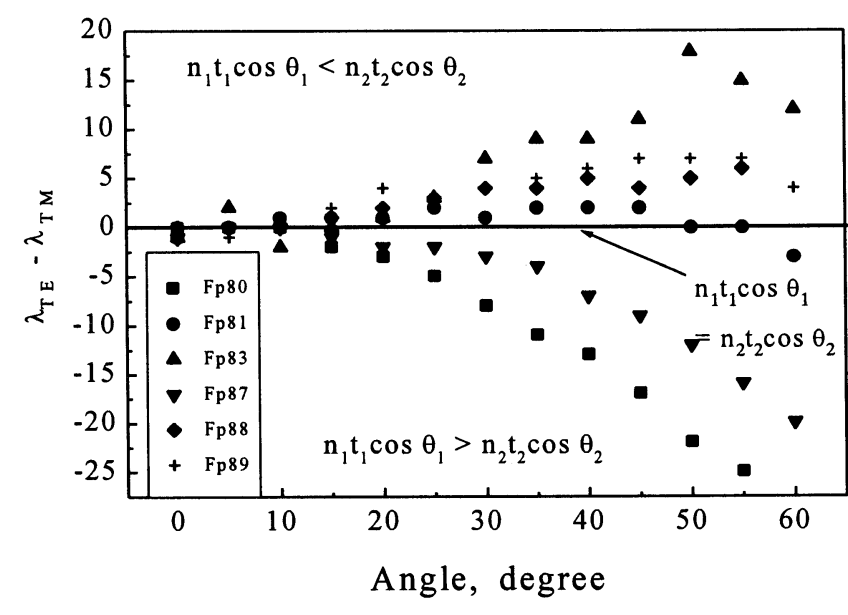

Fig. 8. Spectral difference $\Delta \lambda$ as a function of detection angle for six microcavities all centred at $800 \mathrm{~nm}$. The ratio $\delta$ of the layers constituting the DBR is given for each sample. All samples have 5.5 periods for the surface DBR and 18 periods for the bottom DBR, with $n_{1}>n_{2}$.

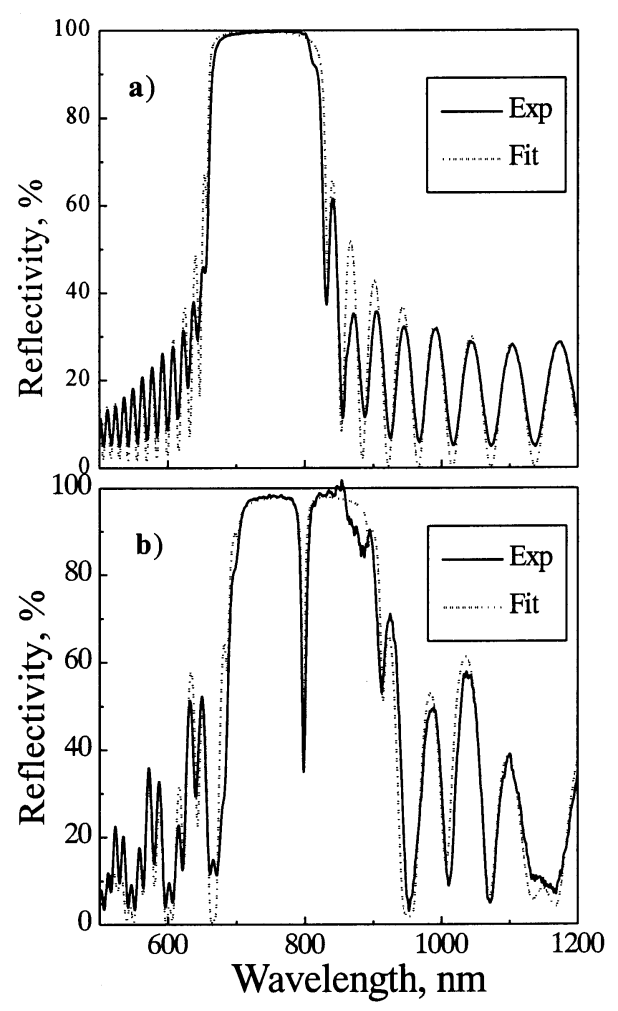

Fig. 9. Fit (dashed line) of the reflectivity spectrum (solid line) of a DBR (a) and a microcavity (b) with each period precisely constituted by two $\lambda / 4$ thick layers. The parameters determined by the fit are $n_{1}=1.9$ and $n_{2}=1.26$, at $\lambda=800 \mathrm{~nm}$ for both samples and $t_{1}=105$ $\mathrm{nm}, t_{2}=158 \mathrm{~nm}$, for the microcavity and $t_{1}=99 \mathrm{~nm}, t_{2}=147 \mathrm{~nm}$ for the DBR.

Using this series of measurements we were able to obtain the formation parameters necessary to realise microcavities having both individual thin layers of the DBR exactly equal for detection normal to the surface.
With these considerations we were able to fit the experimental reflectivity spectra of some DBR and microcavities, as shown in Fig. 9. The fit is in good agreement with the experimental data in a $800 \mathrm{~nm}$ wide range. The refractive indices and the thicknesses determined with the fit are also in agreement with the ratio of the optical thicknesses obtained from the angular dependence of the peak position.

\subsection{Microcavities filled with dye}

In addition to its strong luminescence, PSi has the interesting property of being a good matrix for other emitting materials. In this way we can obtain PSi-based structures that emit at wavelengths that are outside the PSi emission spectrum. In our case we used the Rhodamine 800 (LD800) dye as the emitting material. Rhodamine 800 is a planar organic molecule whose total length is smaller than $1 \mathrm{~nm}$ and is composed of seven benzene cycles. Thanks to its structure the molecule has an absorption spectrum in the 550-750 $\mathrm{nm}$ range with a maximum at $680 \mathrm{~nm}$. The emission spectrum is in the $650-950 \mathrm{~nm}$ range. This organic molecule needs to be sufficiently dispersed to have good luminescence efficiency. We already proved [22] that this dye fills homogeneously the PSi matrix. In Fig. 10 we show a PL spectrum of a filled microcavity (dashed line) compared with the luminescence of a filled single PSi layer (solid line) of the same thickness as the microcavity $(7 \mu \mathrm{m})$. The reference layer has a porosity that is an average of the porosity of the layers constituting the microcavity. As it is possible to see in Fig. 10 there is a sharpening of the dye emission spectrum and an amplification of the luminescence by an unexpected factor of 80 at $710 \mathrm{~nm}$. This factor has been obtained

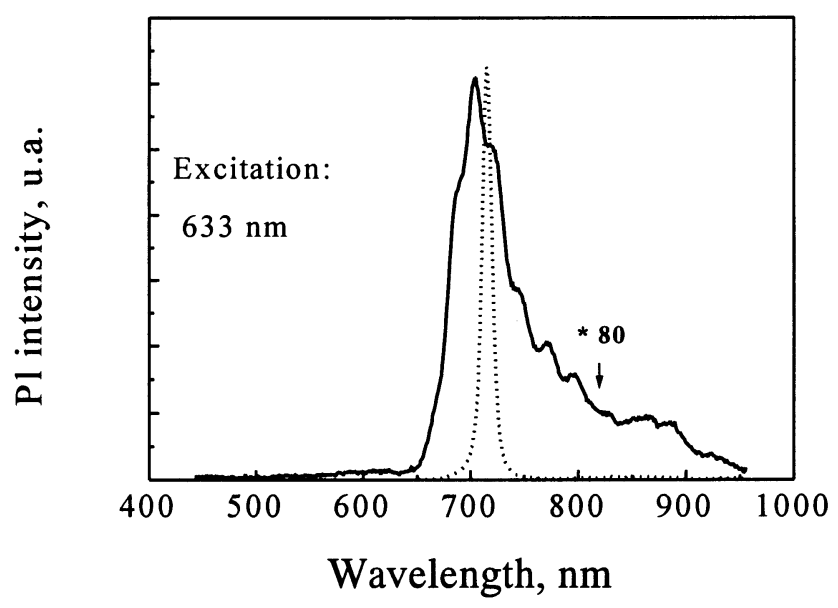

Fig. 10. Room temperature PL spectra of a filled dye microcavity compared to that of a reference layer. In order to compare the various PL lineshapes, the emission of the reference layer has been multiplied by a factor 80 . The excitation was the $633-\mathrm{nm}$ HeNe laser line. 
a)

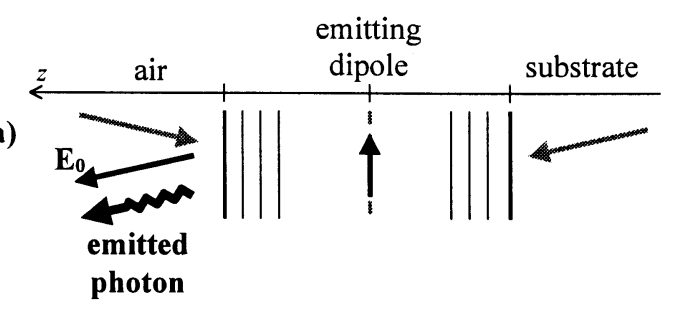

b)

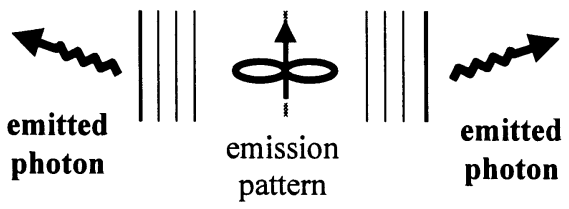

Fig. 11. Sketch of a layered structure with an emitting dipole (e.g. in plane) inside, describing two methods using standard transfer-matrix formalism for the calculation of the emission. (a) Shows the quantum-mechanical approach from [23]: in an 'outgoing' vacuum field mode, one can calculate the field at the location of the emitter, and thus determine its spontaneous emission rate. (b) Describes the classical method from [24]. The classical dipole emits a 'bare' pattern (e.g. here in TM polarisation). The emission is obtained by computing its propagation in the structure.

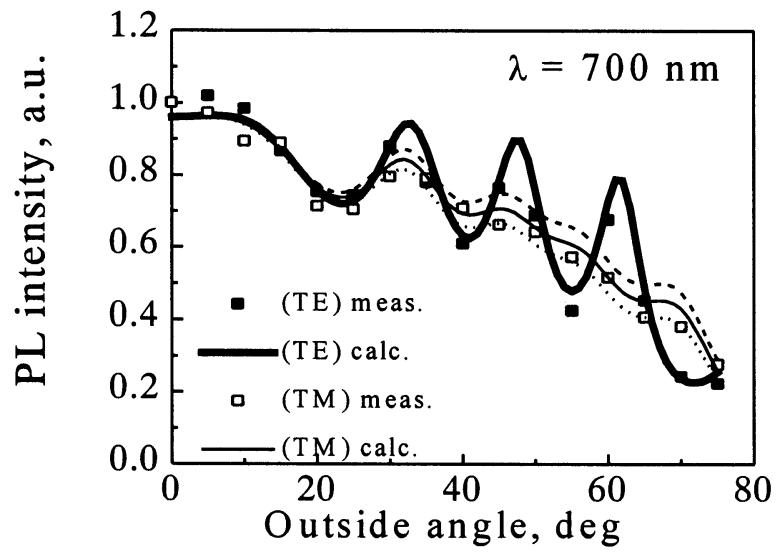

Fig. 12. PL intensity of a PSi single layer $(t=3.11 \mu \mathrm{m}, n=1.30+$ $0.001 \mathrm{i}$ ), measured (squares) and calculated (solid lines) as a function of the emission angle, at $\lambda=700 \mathrm{~nm}$, for both TE and TM polarisations. The calculations are made assuming dipoles homogeneously distributed inside the layer and along the three directions of space. The dashed (respectively dotted) line shows the TM PL intensity, if the number of dipoles perpendicular to the plane ( $z$-dipoles) is $20 \%$ larger (respectively smaller) compared to the number of dipoles in plane.

for a particular excitation angle, which puts the cavity resonance in coincidence with the pumping laser wavelength thus multiplying the expected enhancement of the emission by a simultaneous increase in the excitation.

After introduction of the dye molecule in the microcavity structure, the increase in the losses due to the absorption of the dye produces a decrease in the cavity quality factor $Q=\Delta \lambda / \lambda$ from 150 to 50 , where $\Delta \lambda$ is the FWHM of the cavity mode and $\lambda$ the resonance wavelength.

\subsection{Emission calculations}

Emission in multilayered structures is usually calculated by the method detailed by Björk et al. in [23]. It is based on a quantum-mechanical approach, considering a mode of vacuum field fluctuations (Fig. 11a), and the spontaneous emission rate is given by the Fermi's golden rule. This method is rigorous, but it doesn't permit the calculations in materials with absorption. The first problem is that the vacuum field fluctuations are not classical waves, and the influence of the absorption on them isn't well known. The second problem appears because this calculation needs time reversibility, which is not allowed in the absorption processes.

A more intuitive method is described by Benisty et al. [24]. It considers a classical dipole (Fig. 11b) that emits a wave as if it was in an infinite medium. It also uses the standard transfer-matrix formalism, but the dipole emission is introduced by adding explicit source terms, as a discontinuity of the local field. The main advantage of this method is that it permits us to take into account the absorption in our structures.

In order to check the anisotropy of the emission inside the PSi, the PL of a single layer has been studied as a function of the angle. The PL intensity at $\lambda=700$ $\mathrm{nm}$ as a function of the angle is drawn on Fig. 12. We also show the calculated emission.

For both polarisations, the intensity level, the contrast and the position of the interference fringes show a very good agreement between measurements and calculations. The measurements at large angles in TM polarisation allow us to evaluate the number of $z$-dipoles, compared to the number of dipoles in the plane. On

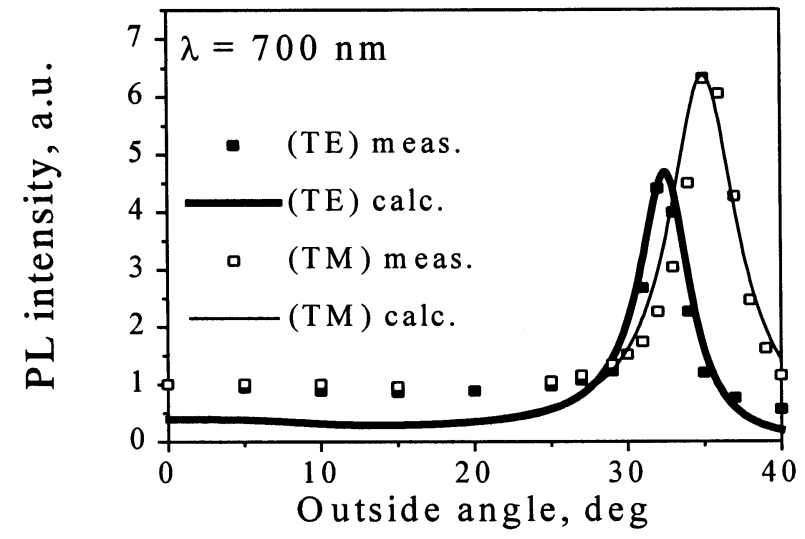

Fig. 13. PL intensity measured (squares) and calculated (solid lines) as a function of the emission angle, at $\lambda=700 \mathrm{~nm}$, for both TE and TM polarisations on a PSi microcavity. The spacer $\left(n_{\mathrm{S}}=1.26+0.01 i\right.$, $\left.t_{\mathrm{S}}=250 \mathrm{~nm}\right)$ is between two distributed Bragg reflectors (5.5 and 18 periods, with $n_{1}=1.75+0.01 i, t_{1}=132 \mathrm{~nm}, n_{2}=1.26+0.01 i, t_{2}=$ $127 \mathrm{~nm}$ ). Calculations are performed assuming a homogeneous distribution of emitting dipoles into the spacer and along the three directions of space. 
Fig. 12, the thin solid line shows the result of the calculation of the PL intensity assuming dipoles homogeneously distributed along the three directions of space. Dashed and dotted lines represent what we may measure if it is not the case.

Measurements show that the difference between the number of dipoles on $z$-direction and in plane does not exceed $20 \%$. In spite of the preferential direction of etching $z$, the distribution of dipoles is relatively isotropic. This is in agreement with the observations made by Kovalev et al. [25]. Their measurements of PL polarisation, with excitation and detection on a cleaved edge of a sample show a ratio of intensities in both polarisations of less than 1.2. In our framework, it could be represented to a reasonable approximation by the same number of dipoles along the three directions.

The most interesting measurements of PL intensity are done on PSi microcavities, because the multilayered structure modifies both the spectral and angular distribution of the light emitted by the material. Fig. 13 shows the PL intensity at $\lambda=700 \mathrm{~nm}$, as a function of the angle for both polarisations.

At this wavelength, from 0 to $30^{\circ}$, the stop band inhibits the emission, and a weak PL intensity is observed. Measurements show that the emission is strongly localised in narrow lobes at $33^{\circ}$ (FWHM $=$ $\left.3.9^{\circ}\right)$ for $\mathrm{TE}$ and $36^{\circ}\left(\mathrm{FWHM}=5.3^{\circ}\right)$ for $\mathrm{TM}$. The two polarisations emit at different angles, because there is no angle for which the optical thicknesses of the different layers of the DBR are rigorously equal to $\lambda / 4$, as explained in the previous discussion. Calculations show a very good agreement with these measurements. Assuming the same absorption effects in all layers $(\operatorname{Im}(n)=0.01)$, the properties of the two peaks are perfectly reproduced, with the same set of parameters for both polarisations.

At low angles, the weak PL intensity measured is due to the emission of the high porosity layers of index $n_{2}$ constituting the DBR and that have the same properties of luminescence than the spacer. The emission of the shallow layers is not efficiently filtered, due to the low number of periods above them.

\section{Conclusion}

In this work we have presented a detailed study on multilayered PSi. Two main issues were investigated: the interface roughness and the optical characterisation. We have shown how the interface roughness can be controlled and that it is then possible to realise very high quality multilayered PSi structures. A new way to determine the highest reflection coefficients is proposed by using CRDS measurements. Moreover, by fitting the spectral position of the cavity resonance, we were able to derive precise information on the main parameters of the structures, like the relative optical thicknesses of the layers composing a DBR. These results opened the way to the realisation of simulations of the reflectivity and luminescence spectra in a very good agreement with the experimental data. In particular, using the Benisty model we were able to fit, taking into account the optical losses, the behaviour of the emission of monolayers and microcavities as a function of the detection angle.

\section{Acknowledgements}

We would like to thank D. Romanini for suggestions and helping in realising CRDS measurements.

\section{References}

[1] L.T. Canham, Appl. Phys. Lett. 57 (1990) 1046.

[2] C. Pickering, M.J. Beale, D.J. Robinson, P.J. Pearson, R. Greef, J. Phys. C: Solid State Phys. 17 (1984) 6535.

[3] G. Vincent, Appl. Phys. Lett. 64 (1994) 2367.

[4] L. Pavesi, Riv. Nuovo Cimento 20 (1997) 1.

[5] G. Lérondel, R. Romestain, Appl. Phys. Lett. 74 (19) (1999) 2740.

[6] M.G. Berger, R. Arens-Fischer, M. Thönissen, M. Krüger, S. Billat, H. Lüth, S. Hilbrich, W. Theiss, P. Grosse, Thin Solid Films 297 (1997) 237.

[7] T.M. Benson, H.F.Arrand, P. Sewell, A. Loni, R.J. Bozeat, M. Krüger, R. Arens-Fischer, M. Thönissen, H. Lüth, Mat. Sci. Eng. B, in press.

[8] L. Pavesi, P. Dubos, Semicon. Science Technol. 12 (1997) 570.

[9] M.G. Berger, R. Arens-Fischer, M. Thönissen, M. Krüger, S. Billat, H. Lüth, S. Hilbrich, W. Theiss, P. Grosse, Thin Solid Films 297 (1997) 237.

[10] D. Hunkel, R. Butz, R. Arens-Fisher, M. Marso, H. Lüth, J. Lum. 80 (1998) 133.

[11] L. Pavesi, C. Mazzoleni, A. Tredicucci, V. Pellegrini, Appl. Phys. Lett. 67 (1995) 3280.

[12] M. Araki, H. Koyama, N. Koshida, Proceedings of the International Symposium on Advanced Luminescent Materials, Electrochemistry Society, Pennington, NJ, USA, 1996, p. 495.

[13] G. Mattei, A. Marucci, V.A. Yakovlev, Mat. Sci. Eng. B 51 (1998) 158.

[14] A. Fainstein, B. Jusserand, V. Thierry-Mieg, Phys. Rev. Lett. 75 (1995) 3764.

[15] L.A. Golovan, A.M. Zheltikov, P.K. Kashkarov, N.I. Koroteev, M.G. Lisachenko, A.N. Naumov, D.A. SidorovBiryukov, V.Y. Timoshenko, A.B. Fedotov, JETP Lett. 69 (1999) 300.

[16] H. Davies, Proc. Inst. Elec. Eng. 101 (1954) 209.

[17] H.E. Bennett, J.O. Porteus, J. Opt. Soc. Am. 51 (1960) 123.

[18] S. Setzu, G. Lérondel, R. Romestain, J. Appl. Phys. 84 (6) (1998) 3129.

[19] D.R. Turner, J. Electrochem. Soc. 105 (7) (1957) 402.

[20] J.N. Chazalviel, R.B. Wehrspohn, F. Ozanam, Mat. Sci. Eng. B (1999), in press.

[21] A. O'Keefe, D.A.G. Deacon, Rev. Sci. Instrum. 59 (1988) 2544. 
[22] S. Setzu, P. Solsona, S. Létant, R. Romestain, J.C. Vial, Eur. Phys. J. A.P. 7 (1999) 59.

[23] G. Björk, Y. Yamamoto, in: H. Yokoyama, K. Ujihara (Eds.), Spontaneous Emission and Laser Oscillations in Microcavities, CRC Press, London, 1995, p. 189.
[24] H. Benisty, R. Stanley, M. Mayer, J. Opt. Soc. Am. A 15 (5) (1998) 1192.

[25] D. Kovalev, M. Ben Chorin, J. Diener, F. Koch, Al.L. Efros, M. Rosen, N.A. Gippius, S.G. Tikhodeev, Appl. Phys. Lett. 67 (11) (1995) 1585. 